\title{
Hydrogen Instability Induced by Postannealing on Poly-Si TFTs
}

\author{
Chia-Chun Liao, Min-Chen Lin, and Tien-Sheng Chao
}

\begin{abstract}
This brief investigates hydrogen instability induced by postannealing. Results show that using a SiN capping layer can prevent the release of hydrogen from a polycrystalline-silicon channel. However, removing this SiN capping layer allows the hydrogen release during postannealing, and the resulting device performance becomes comparable to that of the control sample. Hydrogen release reduces the immunity of PBTI and NBTI. Two possible mechanisms can explain the increased preexisting defects associated with hydrogen release, which affects the NBTI and PBTI.
\end{abstract}

Index Terms-Hydrogen release, NBTI, PBTI, plasma discharge, postannealing.

\section{INTRODUCTION}

$\mathbf{P}$ OLYCRYSTALLINE silicon (poly-Si) thin-film transistors (TFTs) have attracted attention recently, due to their potential applications in active-matrix liquid crystal displays and high-density vertical components [1]. Because the grain boundaries in poly-Si TFTs have a profound influence on device characteristics and degrade carrier transport, developing promising techniques to reduce the trap density is important. Previous research shows that hydrogen-plasma treatment and hydrogen-containing-nitride film deposition are promising approaches for improving performance [2], because hydrogen radicals can effectively passivate the defects of intragrain, grain boundary, and the gate dielectric.

In addition to using hydrogen plasma or hydrogen-containing nitride to passivate the traps deliberately, the hydrogen diffuses into the polysilicon during the fabrication process, in areas such as the buffered layer, the gate dielectric, and the hard mask of nanowires [3]. However, this hydrogen diffusion into the polysilicon easily suffers from subsequent thermal processes such as solid-phase crystallization, the deposition of gate dielectric, and the dopant activation, resulting in unavoidable hydrogen instability.

Postannealing decreases the benefits of using hydrogen radicals by plasma treatment to passivate these defects, indicating that there are limits to thermal cycling after the hydrogenation process. The effects of the postannealing temperature on

Manuscript received August 30, 2011; accepted March 13, 2012. Date of publication April 17, 2012; date of current version May 23, 2012. This work was supported by the National Science Council, Taiwan, under Contract NSC 97-2221-E-009-152-MY3. The review of this brief was arranged by Editor M. J. Kumar.

The authors are with the Department of Electrophysics, National Chiao Tung University, Hsinchu 300, Taiwan (e-mail: tschao@mail.nctu.edu.tw).

Color versions of one or more of the figures in this paper are available online at http://ieeexplore.ieee.org.

Digital Object Identifier 10.1109/TED.2012.2191411 the poly-Si-TFT performance have also been studied experimentally [4]. However, the effects of the hydrogen instability induced by postannealing on the passivation of tail states, the passivation of deep states, and the additional defect generation in poly-Si TFTs must be clarified. To investigate the effects of hydrogen instability, it is necessary to eliminate the $\mathrm{Si}-\mathrm{H}$ bonds completely by postannealing and avoid the issue of plasma damage.

In this brief, this study investigates the passivation efficiency of hydrogen using hydrogen-containing nitride. Although the issue of hydrogen has been thoroughly investigated for singlecrystal MOSFETs [5], to our knowledge, this is the first time the issue of hydrogen release for poly-Si-TFT capped $\mathrm{SiN}$ has been demonstrated. These results indicate two types of defects: the original type of defect affected by passivation/depassivation and a second type generated by hydrogen release during subsequent annealing.

\section{EXPERIMENTAL PROCEDURE}

An amorphous-silicon film measuring $500 \AA$ thick was deposited on thermally oxidized wafers at $500{ }^{\circ} \mathrm{C}$ by a lowpressure chemical vapor deposition (LPCVD). The wafers were annealed at $600{ }^{\circ} \mathrm{C}$ for $24 \mathrm{~h}$ to transform the amorphous film into poly-Si, and the poly-Si films were patterned into active islands. After removing the surface oxide, a 30-nm-thick tetraethyl orthosilicate (TEOS) oxide was deposited on the wafers. A 2000- $\AA$-thick poly-Si was deposited and patterned to form the gate electrode, which also served as a mask for selfaligned implantation. The source/drain and gate regions were then implanted with phosphorous $\left(15 \mathrm{keV}\right.$ at $\left.5 \times 10^{15} \mathrm{~cm}^{-2}\right)$ and activated at $600{ }^{\circ} \mathrm{C}$ for $24-\mathrm{h}$ annealing in $\mathrm{N}_{2}$ ambient. Then, a 10-nm-thick TEOS oxide was deposited. Furthermore, a 200-nm SiN was deposited by LPCVD at $780{ }^{\circ} \mathrm{C}$ for coupling hydrogen without plasma damage. During the deposition process, the hydrogen radicals diffused and passivated the defects in the channel. Under the same pressure, all samples received the same thermal budget by annealing in the nitrogen. The sample, denoted as SiN-removed, was capped by a $10-\mathrm{nm}$ TEOS oxide and a 200-nm SiN layer originally. The capping SiN was then removed by a carefully selective wet-etching process. A 500-nm-thick TEOS oxide formed at $680{ }^{\circ} \mathrm{C}$ was adopted as the passivation layer as well as postannealing for all samples. After patterning the contact holes, aluminum was deposited by PVD and patterned as the probe pads to complete the TFT devices. Poly-Si TFTs of different channel widths from 10 to $0.8 \mu \mathrm{m}$ and gate lengths from 10 to $1 \mu \mathrm{m}$ were fabricated to analyze the passivation effects. 

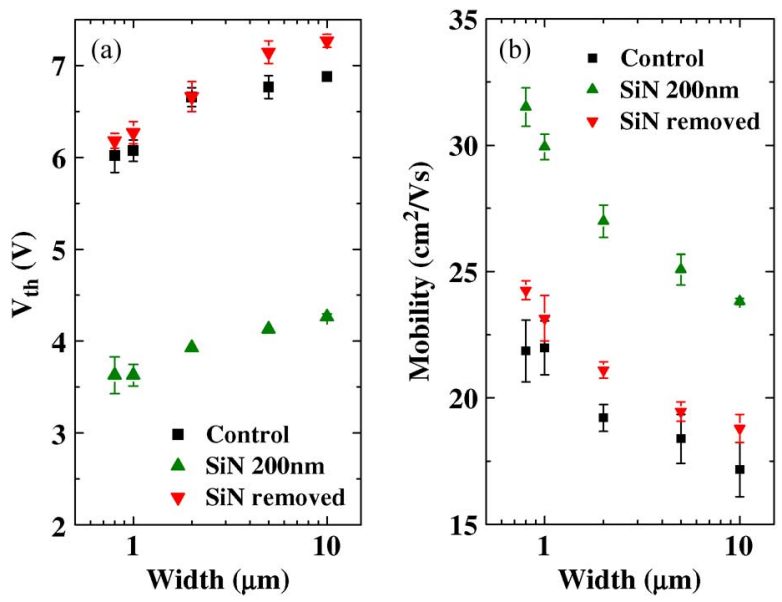

Fig. 1. (a) Variation of threshold voltage as a function of channel width with $L=10 \mu \mathrm{m}$ for the control sample, the nitride-capped sample, and the nitrideremoved sample. (b) Variation of mobility as a function of channel width with $L=10 \mu \mathrm{m}$ for the control sample, the nitride-capped sample, and the nitrideremoved sample.

\section{Results AND Discussion}

\section{A. Hydrogen Instability Induced by Postannealing}

Fig. 1(a) and (b) demonstrates the threshold voltage and mobility as a function of width. The threshold voltage $\left(V_{\mathrm{th}}\right)$ is defined as the gate voltage required to achieve a normalized drain current of $I_{D}=(\mathrm{W} / \mathrm{L}) \times 100 \mathrm{nA}$ at $V_{\mathrm{DS}}=0.1 \mathrm{~V}$. The threshold voltage of the control sample decreases as the channel widths fall from 10 down to $0.8 \mu \mathrm{m}$, since the gate control is further increased with decreasing channel width due to corner-portion domination [1]. The field-effect mobility $\mu_{\mathrm{FE}}$ is extracted from the maximum transconductance. It is noted that the passivation effect by PECVD SiN passivation layer or plasma discharge exhibits strong width dependence, since the portion of the poly-Si channel without passivation by radicals is minimized as the channel width is scaled down [2]. The improvements in threshold voltage and mobility by capping LPCVD SiN are significant for all width dimensions, implying that the LPCVD SiN passivation layer exhibits higher passivation efficiency.

The SiN-removed sample reveals threshold voltage and mobility characteristics comparable to those of the control sample. It is noted that the deep states, which originate from the dangling bonds in the grain boundaries, influence the threshold voltage and the subthreshold swing. However, tail states, which originate from the intragrain defects, affect the field-effect mobility and the minimum leakage current [2]. This implies that postannealing breaks both the passivation of the dangling bonds and the intragrain defects by hydrogen, which in turn decreases the improvement in threshold voltage and mobility. By the same token, the comparison shows that the SiN capping layers can strengthen the immunity against the postannealing, due to the suppression of hydrogen outgassing during postannealing.

Fig. 2 shows the drain-current-versus-drain-voltage $\left(I_{D}-V_{D}\right)$ characteristics of all n-channel poly-Si TFTs. The SiN-200-nm sample exhibits a significantly better performance than the control sample due to a reduction in the tail state density. The SiN-removed sample exhibits a performance comparable to that

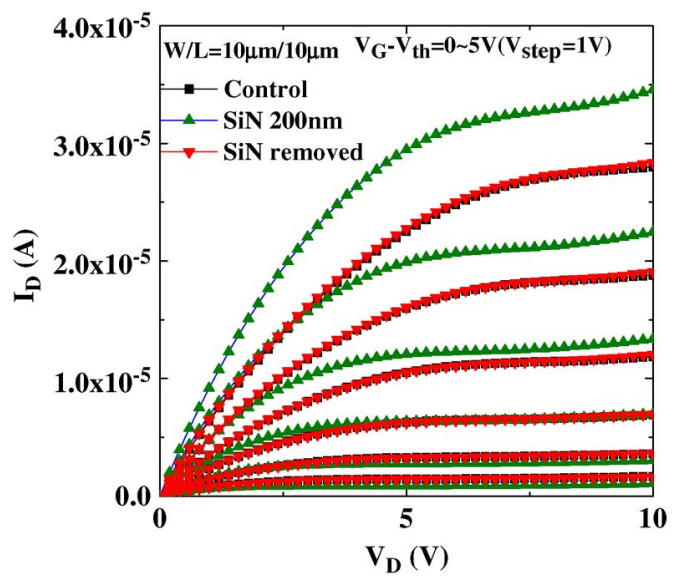

Fig. 2. Output characteristics (the $I_{D^{-}} V_{D}$ curve) of n-channel TFTs with $W / L=10 \mu \mathrm{m} / 10 \mu \mathrm{m}$ for the control sample, the nitride-capped sample, and the nitride-removed sample.

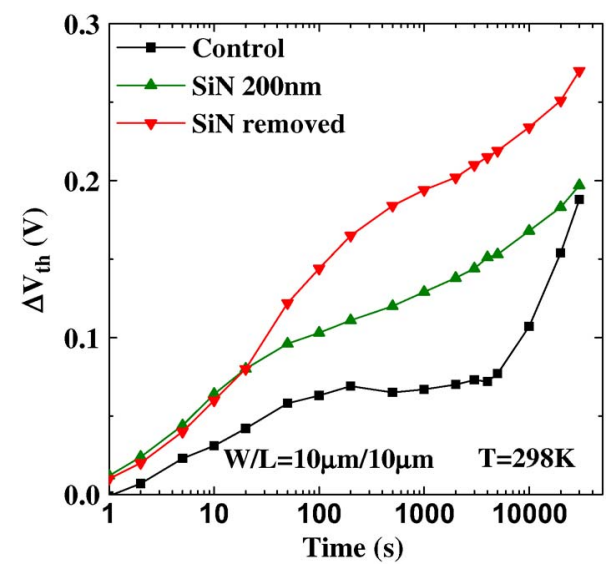

Fig. 3. Threshold-voltage shift as a function of stress time for the control sample, the nitride-capped sample, and the nitride-removed sample with $W / L=10 \mu \mathrm{m} / 10 \mu \mathrm{m}$ under positive gate bias stress as $V_{\mathrm{GS}}=19 \mathrm{~V}$.

of the control sample, indicating that the $\mathrm{Si}-\mathrm{H}$ bonds, which affect the electrical properties, break completely.

Fig. 3 shows the time dependence of threshold-voltage shift $\Delta V_{\text {th }}$ of the n-channel TFTs after the PBTI stress with $V_{G}=$ $19 \mathrm{~V}$ and $V_{D}=V_{s}=0 \mathrm{~V}$ for $30000 \mathrm{~s}$ at $T=25{ }^{\circ} \mathrm{C}$. BTI degradation was evaluated by the conventional method in which $I_{D}-V_{G}$ curves are measured during stress interruption. It can be seen that the increase in the $\Delta V_{\mathrm{th}}$ of the SiN-removed sample is higher than that of the TEOS-10-nm and control samples, implying a raised defect density.

Fig. 4 shows the time dependence of the threshold-voltage shift $\Delta V_{\text {th }}$ of the n-channel TFTs after the NBTI stress with $V_{G}=-19 \mathrm{~V}$ and $V_{D}=V_{s}=0 \mathrm{~V}$ for $30000 \mathrm{~s}$ at $T=25^{\circ} \mathrm{C}$. The control sample and SiN-200-nm sample exhibit an increasing threshold voltage after NBTI stressing, but the SiN-removed sample exhibits a decreasing threshold voltage. Furthermore, the insignificant degradation of the subthreshold swing indicates insignificant negatively charged $\Delta Q_{\text {it }}$ (not shown here). Thus, the different dominant charge trappings are responsible for the different immunities of the NBTI, implying different oxide quality and channel conditions. As a result, the control sample and the SiN-removed sample exhibit different 


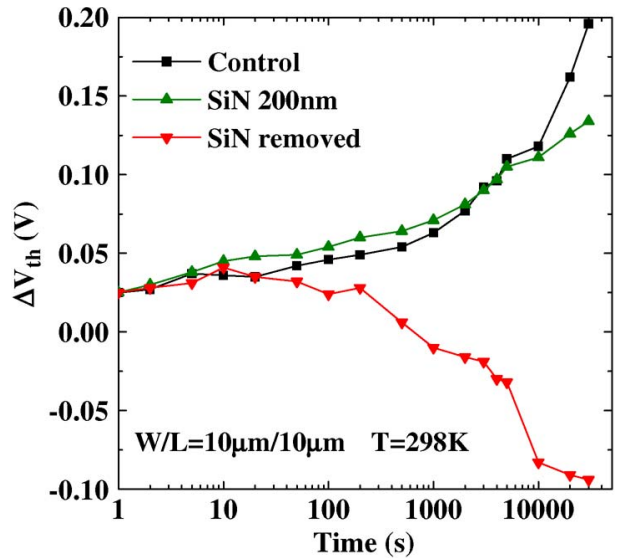

Fig. 4. Threshold-voltage shift as a function of stress time for the control sample, the nitride-capped sample, and the nitride-removed sample with $W / L=10 \mu \mathrm{m} / 10 \mu \mathrm{m}$ under negative gate bias stress as $V_{\mathrm{GS}}=-19 \mathrm{~V}$.

immunities to NBTI stressing, although their original performances are comparable. It is known from the literature that the interface between the poly-Si channel and gate oxide exhibits better quality than the interface between the poly-Si gate and gate oxide [6]. The nonideal interface between the poly-Si gate and gate oxide results in the significant electron injection from the poly-Si gate under negative bias stressing. On the other hand, the hydrogen release degrades the interface between the poly-Si channel and gate oxide, and the hole injection from the poly-Si channel becomes higher than the electron injection from the poly-Si gate, as a result.

Based on our results, there are two possible mechanisms for the increase in preexisting defects in the SiN-removed sample. The first mechanism is the hydrogen or hydroxide group, which does not affect the original performance as strongly as the control sample, inducing traps, preexistent to the stress or created during the stress in the gate oxide [7]. The second possible cause is the out diffusion of hydrogen released from the intragrain or grain boundary, since such hydrogen migration may generate defects, such as platelets, $\mathrm{H}_{2}$ defects, and vacancies in the poly-Si channel [8].

\section{CONCLUSION}

This brief has investigated the effects of hydrogen instability induced by postannealing on device performance and reliability. For the SiN-removed sample, postannealing decreases the improvements of defect passivation. Devices with a $\mathrm{SiN}$ capping layer can suppress the outgassing of hydrogen while retaining the improvement in mobility and threshold voltage.

Hydrogen release reduces the immunities of PBTI and NBTI. Samples experiencing hydrogen release have exhibited the worst degradation of PBTI. For NBTI concerns, the dominant degradation mechanism of the threshold voltage of the SiNremoved sample has been the hole trapping rather than the electron trapping, which has been the mechanism that affects both the control sample and the capped SiN sample. Two possible mechanisms can explain the increased preexisting defects that affect the NBTI and PBTI.

\section{ACKNOWLEDGMENT}

The authors would like to thank the National Nano Device Laboratories and the Nano Facility Center, National Chiao Tung University, for the process.

\section{REFERENCES}

[1] M. C. Tsai, T. C. Liao, I. C. Lee, and H. C. Cheng, "Passivation-induced subthreshold kink effect of ultrathin-oxide low-temperature polycrystalline silicon thin film transistors," IEEE Electron Device Lett., vol. 32, no. 7, pp. 904-906, Jul. 2011.

[2] C. C. Liao, M. C. Lin, T. Y. Chiang, and T. S. Chao, "Effects of channel width and nitride passivation layer on electrical characteristics of polysilicon thin film transistors," IEEE Trans. Electron Device, vol. 58, no. 11, pp. 3812-3819, Nov. 2011.

[3] C. W. Luo, H. C. Lin, K. H. Lee, W. C. Chen, H. H. Hsu, and T. Y. Huang, "Impacts of nanocrystal location on the operation of trap-layer-engineered poly-Si nanowired gate-all-around SONOS memory devices," IEEE Trans. Electron Devices, vol. 58, no. 7, pp. 1879-1885, Jul. 2011.

[4] F. S. Wang, M. J. Tsai, and H. C. Cheng, "The effects of $\mathrm{NH}_{3}$ plasma passivation on polysilicon thin-film transistors," IEEE Electron Device Lett., vol. 16, no. 11, pp. 503-505, Nov. 1995.

[5] Z. F. Zhang, H. K. Sii, R. Degraeve, and G. Groeseneken, "Mechanism for the generation of interface state precursors," J. Appl. Phys., vol. 87, no. 6, pp. 2967-2977, Mar. 2000.

[6] T. F. Lei, J. Y. Cheng, S. Y. Shiau, T. S. Chao, and C. S. Lai, "Characterization of polysilicon oxides thermally grown and deposited on the polished polysilicon films," IEEE Trans. Electron Devices, vol. 45, no. 4, pp. 912 917, Apr. 1998.

[7] V. Huard, M. Denais, F. Perrier, N. Revil, C. Parthasarathy, A. Bravaix, and E. Vincent, "A thorough investigation of MOSFETs NBTI degradation," Microelectron. Reliab., vol. 45, no. 1, pp. 83-98, Jan. 2005.

[8] F. A. Reboredo, M. Ferconi, and S. T. Pantelides, "Theory of the nucleation, growth, and structure of hydrogen-induced extended defects in silicon," Phys. Rev. Lett., vol. 82, no. 24, pp. 4870-4873, Jun. 1999.

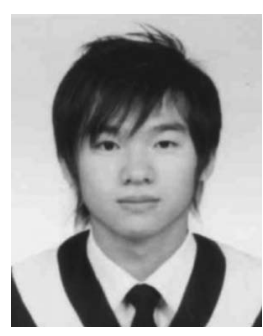

Chia-Chun Liao was born in Taoyuan, Taiwan, in 1980. He received the B.S. degree in electrophysics from National Chiao Tung University, Hsinchu, Taiwan, in 2007. He is currently working toward the Ph.D. degree in the Department of Electrophysics, National Chiao Tung University.

His research interests include strain technique, PEALD for metal gate/high- $k$ application, and nonvolatile flash memories.

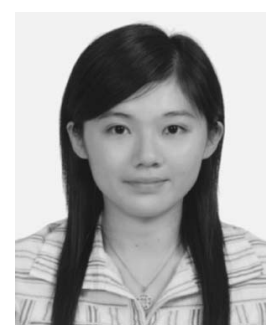

Min-Chen Lin was born in Tainan, Taiwan, in 1986. She received the B.S. degree in physics from the National Cheng Kung University, Tainan, in 2008 and the M.S. degree from the Institute and Department of Electrophysics, National Chiao Tung University, Hsinchu, Taiwan, in 2011.

She is currently with the Department of Electrophysics, National Chiao Tung University. Her research interests are the fabrication and characterization of nonvolatile flash memory and high- $k$ dielectrics.

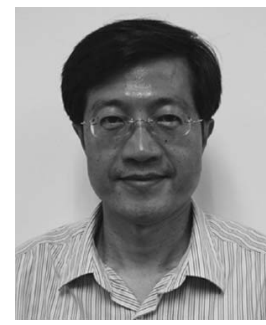

Tien-Sheng Chao was born in Penghu, Taiwan, in 1963. He received the Ph.D. degree in electronics engineering from National Chiao Tung University, Hsinchu, Taiwan, in 1992.

In July 1992, he joined the National Nano Device Laboratories as an Associate Researcher, where he became a Researcher in 1996. He was engaged in developing thin-dielectrics preparations, cleaning processes, and CMOS device fabrication. Then, since 2001, he has been with the Department of Eleche became a Professor in 2002. trophysics, National Chiao Tung University, where 